\title{
Acute Immunotoxic Effects of Perfluorononanoic Acid (PFNA) in C57BL/6 Mice
}

\section{Cheryl E Rockwell ${ }^{1}$, Alexandra E Turley ${ }^{1}$, Xingguo Cheng $^{2}$, Patrick E Fields ${ }^{3}$ and Curtis D Klaassen ${ }^{2 *}$}

${ }^{1}$ Department of Pharmacology \& Toxicology, Michigan State University, East Lansing, MI 48824, USA

${ }^{2}$ Department of Pharmacology, Toxicology \& Therapeutics, University of Kansas Medical Center, Kansas City, KS 66160, USA

${ }^{3}$ Department of Pathology \& Laboratory Medicine, University of Kansas Medical Center, Kansas City, KS 66160, USA

\begin{abstract}
otrganic perfluorochemicals (PFCs) have become an environmental concern due to widespread detection in human blood and experimental evidence for immune, developmental, and liver toxicity. Whereas the blood concentrations of many PFCs are declining, blood levels of Perfluorononanoic Acid (PFNA) are rising in the United States. The purpose of the present studies was to determine the effects of PFNA on lymphoid organs and immune cells of C57BL/6 mice. The present study demonstrates that PFNA produces immunotoxic effects in both male and female C57BL/6 mice as evidenced by splenic atrophy, decreased splenocyte numbers, and a marked reduction in thymocyte viability. The current study also demonstrates that the effects of PFNA on different leukocyte populations are not uniform. The $\mathrm{CD} 4^{+} \mathrm{CD} 8^{+}$double-positive thymocytes were particularly sensitive to PFNA in which the proportion of this population was $>95 \%$ decreased relative to the entire $\mathrm{CD} 4^{+}$thymocyte population in PFNA-treated mice. Interestingly, PFNA also markedly increased serum levels of TNFa in response to LPS in mice. Collectively, the present studies demonstrate that PFNA decreases lymphocyte viability and alters the immune response to LPS in C57BL/6 mice.
\end{abstract}

Keywords: Perfluorononanoic acid; PFNA, Perfluoroalkyl acid; T cell; LPS; TNFa

\section{Introduction}

organic perfluorochemicals (PFCs) are widely used in industry for a variety of applications, including food packaging, flame retardants, waterproof coatings, surfactants as well as in many other products [1-3]. Dozens of PFCs are used in manufacturing, among these are perfluorooctanoic acid (PFOA), perfluorooctane sulfonic acid (PFOS), perfluorononanoic acid (PFNA) and the ammonium salts of these compounds [4]. PFCs have been manufactured since the 1950's, have low volatility, and are persistent in the environment $[1,4]$. The elimination half-lives of PFOA and PFOS in humans are estimated to be from 3 to 5 years $[1,5]$. Whereas the investigation of PFOA and PFOS has been a major area of toxicological research, other PFCs, such as PFNA, are not well characterized in this regard. Thus, in general, the sources, toxicokinetic and potential pathophysiological effects of PFNA in humans and animals necessitate further investigation.

Although most of the PFCs have not been extensively studied, evidence thus far suggests many of these compounds may cause immunological, developmental and liver toxicity $[3,5,6]$. With respect to PFNA, studies have demonstrated that prenatal exposure of mice to PFNA negatively impacts survival and development of pups [7]. PFNA administration also causes male-specific reproductive toxicity in rats, which is attributed to a defect in the secretory function of Sertoli cells $[8,9]$. In vitro studies indicate that PFNA causes only modest DNA damage at high concentrations, suggesting that genotoxicity is not a major concern [10]. Epidemiological data indicate a positive association between human serum levels of PFNA and total cholesterol, suggesting that PFNA exposure may alter cholesterol metabolism [11]. Of relevance to the present studies, daily administration of PFNA over 14 days causes apoptosis in spleens of BALB/c mice, an experimental mouse model of Th2-skewed immune responses [12].

The purpose of the present studies was to ascertain the effect of a single dose of PFNA $(0.1 \mathrm{mmol} / \mathrm{kg})$ on lymphoid organs and immune cell populations of $\mathrm{C} 57 \mathrm{BL} / 6$ mice. The present studies are particularly important given that the immunotoxic effects of PFCs appear to be highly dependent upon mouse strain [6]. Whereas administration of $30 \mathrm{mg} / \mathrm{kg} /$ day of PFOA daily for 15 days causes a marked decrease in weight of lymphoid organs in C57BL/6 mice, Sv/129 mice appear to be relatively insensitive to this effect. With respect to the current study, we are particularly interested in the $\mathrm{C} 57 \mathrm{BL} / 6$ strain because it represents an experimental model that is not Th2-skewed and is also the most commonly-used mouse model in research.

\section{Materials and Methods}

\section{Materials}

PFNA (free acid form; $97 \%$ purity; M.W. $464 \mathrm{~g} / \mathrm{mol}$ ) was purchased from Sigma-Aldrich Co. (St Louis, MO).

\section{PFNA and LPS administration to mice}

Eight-week-old adult male and female C57BL/6J mice were purchased from Jackson Laboratories (Bar Harbor, Maine), and housed according to AAALAC (Association for Assessment and Accreditation of Laboratory Animal Care) guidelines. Adult C57BL/6 male $(\mathrm{n}=5)$ and female $(n=4)$ mice were administered a single i.p. dose of PFNA $(0.1$ $\mathrm{mmol} / \mathrm{kg}$ of body weight), and control mice were treated i.p. with the vehicle, propylene glycol:water $(1: 1, \mathrm{v} / \mathrm{v})$. After two weeks, the spleen, thymus, kidney, and liver were collected from each mouse. Lymph nodes were undetectable (or at least very difficult to distinguish) in PFNA-treated mice. For studies with LPS, LPS was administered two weeks after PFNA exposure. The mice were given a single i.p. dose of LPS or saline ( $1 \mathrm{mg} / \mathrm{kg}$ of body weight, diluted in saline). One hour and thirty minutes after LPS administration, blood was collected. The animal studies were conducted in accordance with the Guide for

*Corresponding author: Curtis D Klaassen, PhD, Department of Pharmacology Toxicology \& Therapeutics University of Kansas Medical Center, 3901 Rainbow Blvd., Kansas City, KS 66160, Tel: 1-913-588-7714; Fax: 1-913-588-7501; E-mail: cklaasse@kumc.edu

Received March 12, 2013; Accepted April 15, 2013; Published April 18, 2013

Citation: Rockwell CE, Turley AE, Cheng X, Fields PE, Klaassen CD (2013) Acute Immunotoxic Effects of Perfluorononanoic Acid (PFNA) in C57BL/6 Mice. Clin Exp Pharmacol S4: 002. doi:10.4172/2161-1459.S4-002

Copyright: (C) 2013 Rockwell CE, et al. This is an open-access article distributed under the terms of the Creative Commons Attribution License, which permits unrestricted use, distribution, and reproduction in any medium, provided the original author and source are credited. 
the Care and Use of Laboratory Animals as adopted by the National Institutes of Health, and were approved by the Institutional Animal Care and Use Committees (IACUC) at the University of Kansas Medical Center and/or Michigan State University.

\section{Cell isolation}

Spleens and thymuses were pulverized, made into single cell suspensions and filtered through nylon mesh. Leukocytes and red blood cells (RBCs) were counted using a hemacytometer (cells were counted in the presence and absence of a RBC-lysing agent).

\section{Flow cytometry}

Freshly-isolated splenocytes were washed and resuspended in FACS buffer (PBS, 1\% FCS). The cells were then incubated with anti-CD4/ PerCP, anti-CD8/APC, anti-CD19/PE, and/or anti-CD14/FITC for 30 $\min$ at $4^{\circ} \mathrm{C}$, after which the cells were washed and fixed with BD Cytofix fixation buffer (BD Biosciences, San Jose, CA). The fluorescence was then detected and quantified with a $\mathrm{BD}^{\mathrm{max}}$ FACSCalibur flow cytometer (BD Biosciences, San Jose, CA). The data were analyzed using FlowJo 7.5 software (Tree Star, Inc., Ashland, OR).

\section{TNF $\alpha$ ELISA}

TNFa levels in serum were quantified using a commercially available mouse TNF- $\alpha$ ELISA kit according to the manufacturer's protocol (Ebioscience, San Diego CA). The relative absorbance of each sample was quantified on a Bio-Tek $\mu$ Quant microplate reader (Highland Park, VT). The TNF- $\alpha$ concentrations were calculated using a 4-parameter standard curve.

\section{Statistical analysis}

The mean \pm standard error was determined for each treatment in the individual experiments. Homogeneous data were evaluated by twoway parametric analysis of variance. When significant differences were observed, the Holm-Sidak post-hoc test was used to compare treatment groups to the vehicle $(\mathrm{VH})$ control using SigmaStat 3.01a software from Systat Software, Inc. (Chicago, IL).

\section{Results}

\section{Splenic atrophy by PFNA}

The immunotoxic effects of PFOA and PFOS have been described in previous studies [6]. To determine the acute effects of PFNA on the immune system, C57BL/6 mice were administered a single dose of PFNA $(0.1 \mathrm{mmol} / \mathrm{kg})$. Effects on lymphoid organs and immune cells were assessed two weeks later. PFNA caused a substantial decrease in body weight in both male (31\%) and female (38\%) mice (Figure 1A). Among the organs examined, the spleen was particularly sensitive to PFNA (with a $60-70 \%$ decrease in spleen to body weight ratio), whereas the decrease in kidney size was proportional to the decrease in overall body weight and thus no difference in the ratio of kidney to body weight was observed with PFNA treatment (Figure 1B and $1 \mathrm{C}$ ). In contrast, PFNA administration resulted in increased liver size $(\approx 300 \%$, Figure 1D).

\section{PFNA decreases leukocyte viability}

In correlation with the decrease in spleen size, a marked decrease in splenic leukocyte counts ( $87.5 \%$ in males, and $\sim 93 \%$ in females) and RBC counts ( $\sim 95 \%$ in males, $\sim 89 \%$ in females, Figure 2 ) was also observed. Altered forward vs. side scatter dot plots were observed in the thymocyte population from PFNA-treated mice (Figure 3). The decrease in forward scatter coupled with the increase in side scatter indicates the emergence of a population of apoptotic cells, which are smaller and denser than untreated lymphocytes.
A.

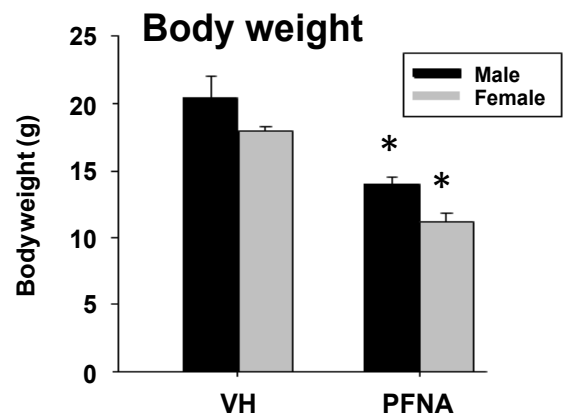

C.

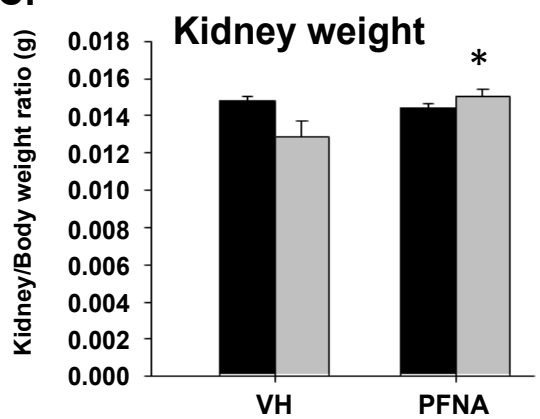

B.

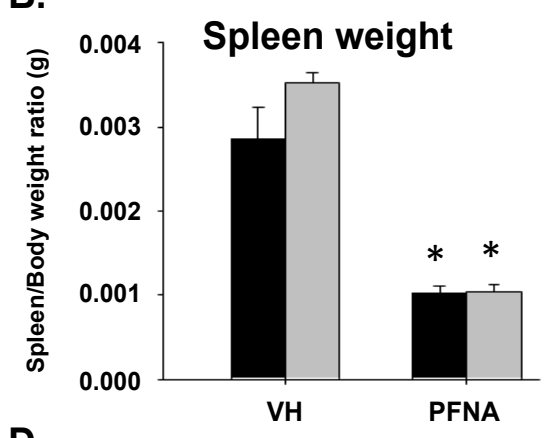

D.

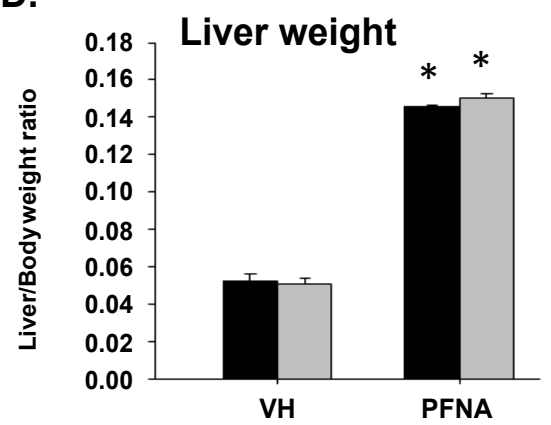

Figure 1: Body and organ weights of mice two weeks after PFNA administration. (A) Body weight and the ratios of (B) spleen, (C) kidney, and (D) liver to body weight were measured two weeks after PFNA administration. The data are presented as the mean \pm SE. * denotes $p<0.05$ as compared to the vehicle $(\mathrm{VH})$ control for the same gender. 

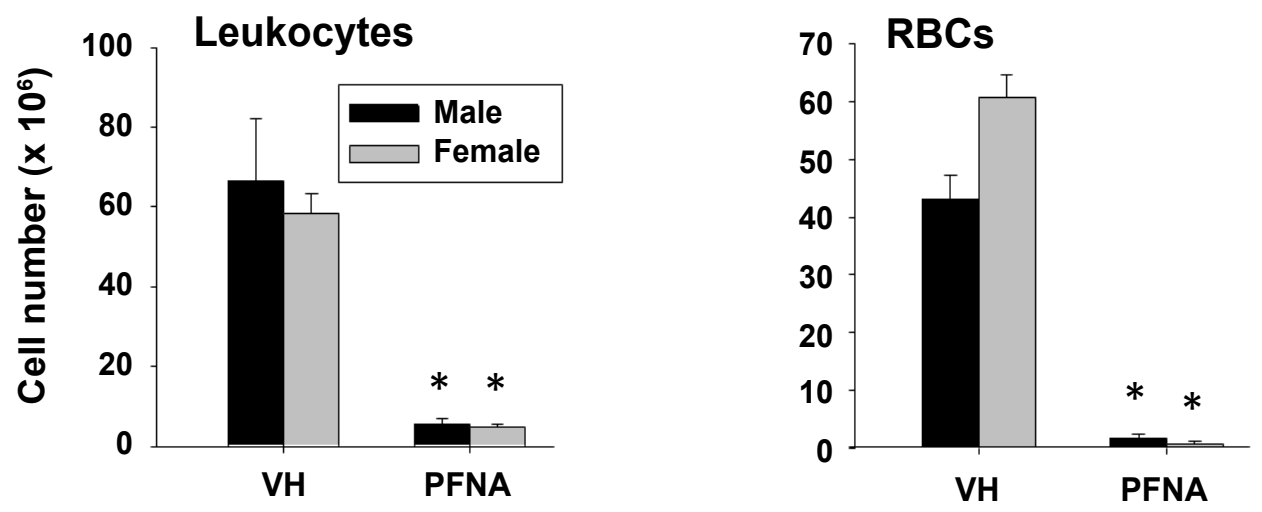

Figure 2: PFNA markedly decreases leukocyte and RBC counts in spleen. Cells were diluted and counted in the presence and absence of an RBC-lysing agent. The data are presented as the mean $\pm \mathrm{SE}$. ${ }^{*}$ denotes $p<0.05$ as compared to the vehicle $(\mathrm{VH})$ control for the same gender.
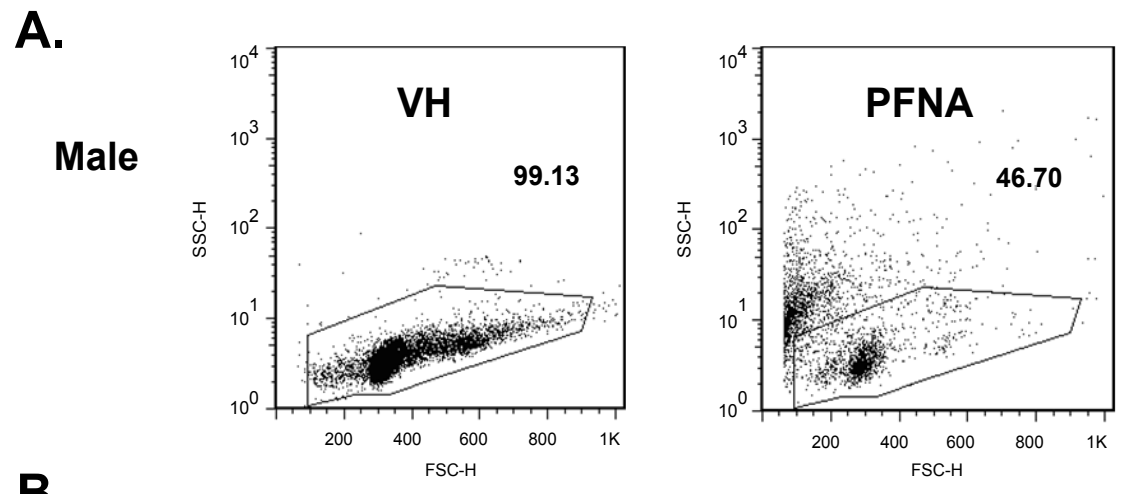

B.
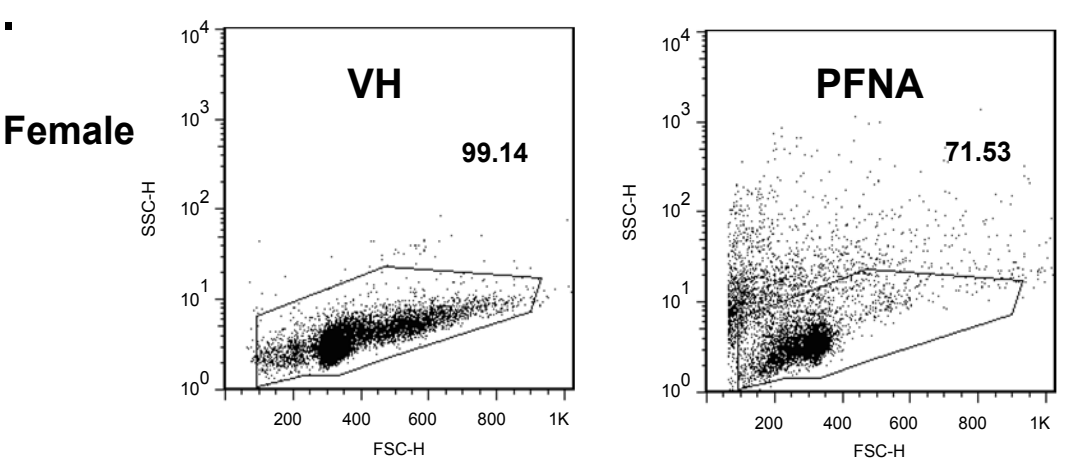

C.

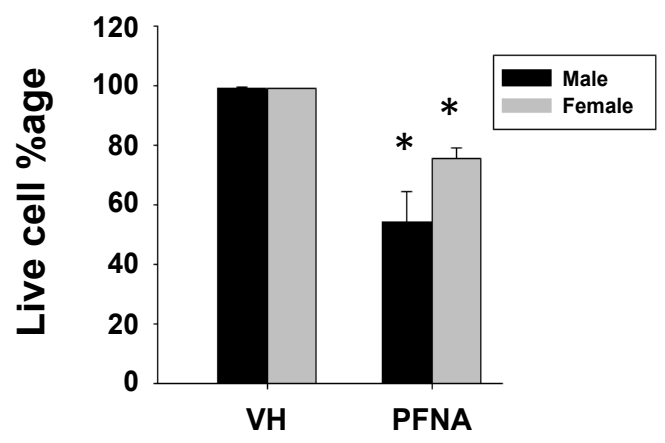

Figure 3: PFNA decreases thymocyte viability as assessed by forward vs. side scatter. Forward vs. side scatter was assessed in (A) male and (B) female thymocytes by flow cytometry. (C) Live cells were quantified and graphed. The data are presented as the mean $\pm \mathrm{SE}$. ${ }^{*}$ denotes $p<0.05$ as compared to the vehicle (VH) control for the same gender. 


\section{Effect of PFNA on splenic T Cells}

To determine the sensitivity of various leukocyte populations to PFNA, we conducted immunophenotyping analysis by flow cytometry. With respect to T cells, PFNA increased the ratios of both $\mathrm{CD} 4^{+}$and $\mathrm{CD}^{+}$cells relative to the entire splenocyte population in female mice (Figure 4). In male mice, PFNA increased the ratio of $\mathrm{CD} 8^{+}$cells and caused a trend toward an increase of $\mathrm{CD} 4^{+}$cells with respect to the entire splenocyte population. Overall, the data suggest $\mathrm{T}$ cells may be slightly less sensitive to the immunotoxic effects of PFNA compared to other splenocyte populations.

\section{PFNA alters immature T Cell populations}

The effect of PFNA on immature T cells in the thymus was also determined. In addition to the marked decrease in viable cells in thymus noted in Figure 3, substantial differences in thymic subpopulations were also observed (Figure $5 \mathrm{~A}$ and $5 \mathrm{~B}$ ). Notably, the $\mathrm{CD} 4^{+} \mathrm{CD} 8^{+}$ double-positive population is largely absent in thymus from both male and female mice treated with PFNA (Figure 5C). With the decrease in the $\mathrm{CD}^{+} \mathrm{CD}^{+}$double-positive population, there was a concurrent increase in the ratios of the $\mathrm{CD} 4^{+}$and $\mathrm{CD} 8^{+}$single-positive populations as well as the CD4CD8 double-negative population (Figure $5 \mathrm{D}-5 \mathrm{~F}$ ) Collectively, the data suggest that although there is a decrease in overall thymocyte viability with PFNA treatment, the $\mathrm{CD} 4^{+} \mathrm{CD} 8^{+}$doublepositive population is particularly sensitive to PFNA.

\section{Effects of PFNA on $\mathrm{CD}_{19}{ }^{+}$and $\mathrm{CD}_{14}{ }^{+}$Cells}

In addition to $\mathrm{CD} 4^{+}$and $\mathrm{CD} 8^{+} \mathrm{T}$ cells, the effect of PFNA on $\mathrm{CD} 19^{+}$ and $\mathrm{CD} 14^{+}$cells was also evaluated. $\mathrm{CD} 19$ is a coreceptor that associates with the B cell receptor and is thus, a commonly-used B cell marker.
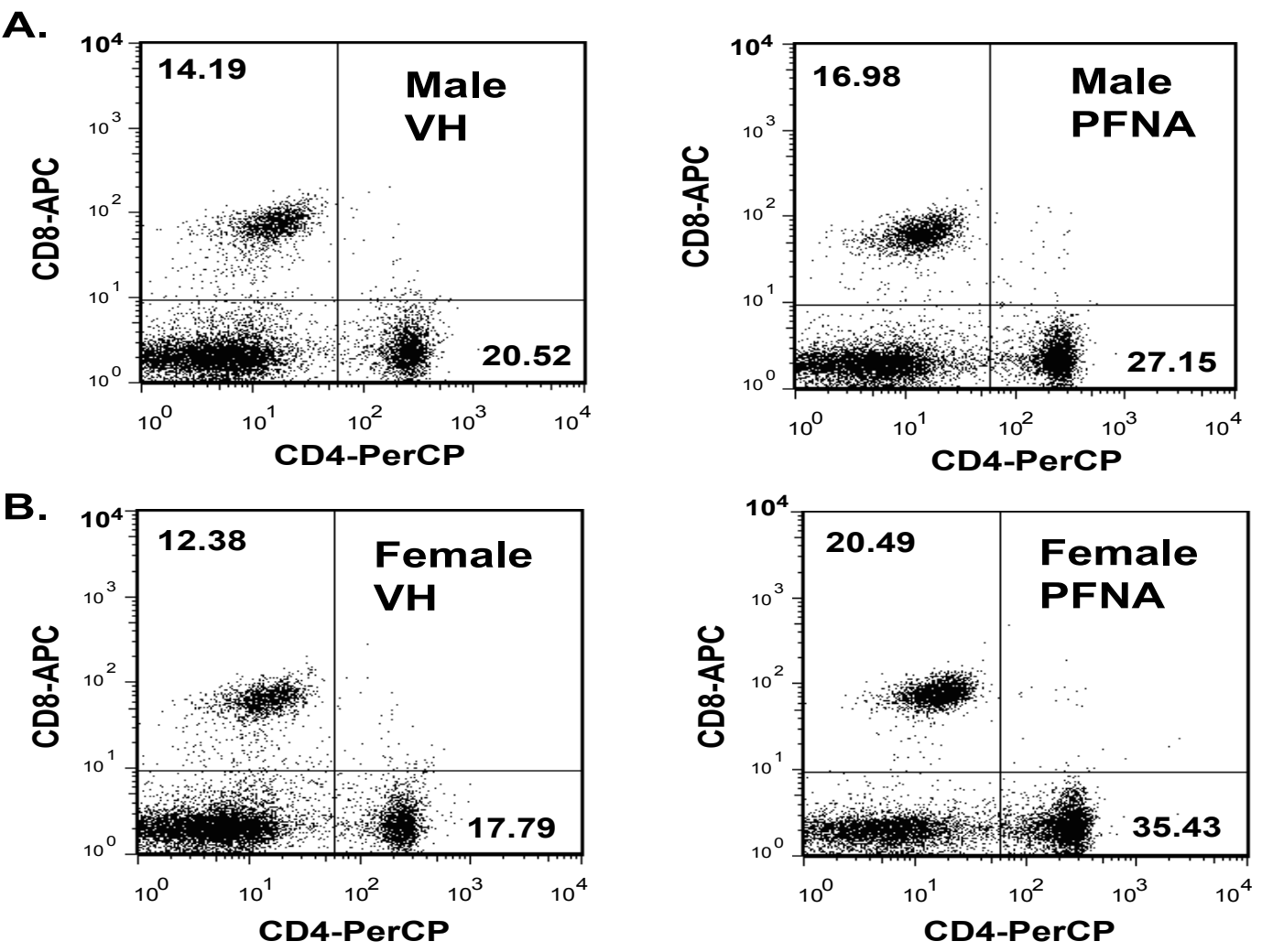

C.

D.
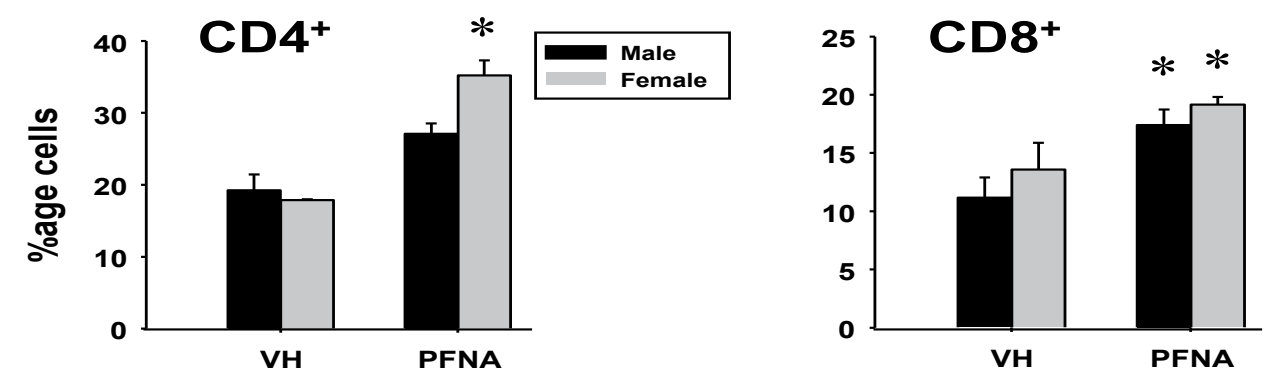

Figure 4: PFNA increases the ratios of $C D 4^{+}$and $C D 8^{+} T$ cells relative to the whole splenocyte population. Unfractionated splenocytes were washed, incubated with fluorochrome-conjugated antibodies and analyzed by flow cytometry. Representative dot plots of CD4 ${ }^{+}$and $\mathrm{CD} 8^{+} \mathrm{T}$ cells are depicted from $\mathrm{VH}$-treated and PFNAtreated $(A)$ male and $(B)$ female mice. The numbers on the dot plot represent the percentage of the population for that quadrant. Graphical representation of $(C)$ $\mathrm{CD}^{+}$and (D) $\mathrm{CD}^{+} \mathrm{T}$ cell percentage with respect to the whole splenocyte population. The data are presented as mean $\pm \mathrm{SE}$. ${ }^{*}$ denotes $\mathrm{p}<0.05$ as compared to the vehicle $(\mathrm{VH})$ control for the same gender. 


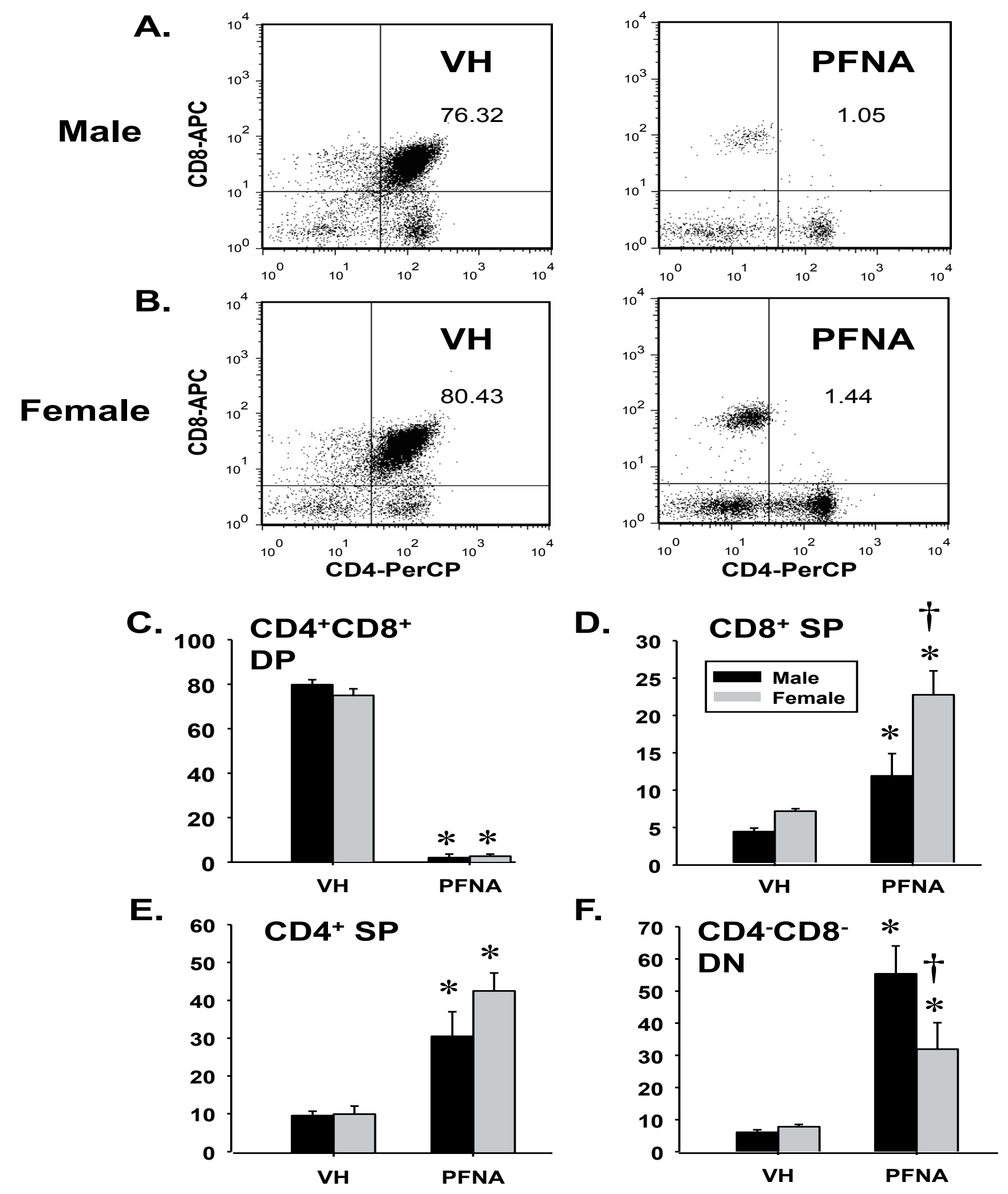

Figure 5: Loss of the $\mathrm{CD} 4^{+} \mathrm{CD} 8^{+}$double-positive $\mathrm{T}$ cell population in thymus of PFNA-treated mice. Unfractionated thymocytes were washed, incubated with fluorochrome-conjugated antibodies and analyzed by flow cytometry. Representative dot plots of $\mathrm{CD}^{+}{ }^{+}$and $\mathrm{CD} 8^{+} \mathrm{T}$ cells are depicted from $\mathrm{VH}$-treated and PFNAtreated (A) male and (B) female mice. Graphical representation of (C) CD4+CD8+ double-positive (DP), (D) CD8+ single-positive (SP), (E) CD4+ SP, (F) CD4-CD8double-negative (DN) T cell percentages with respect to the whole splenocyte population. The data are presented as mean $\pm \mathrm{SE}$. ${ }^{*}$ denotes $\mathrm{p}<0.05$ as compared to the vehicle $(\mathrm{VH})$ control for the same gender. + denotes $p<0.05$ as compared between genders for the same treatment group. 
CD14 is the LPS co-receptor and is used as a marker of phagocytes, such as macrophages and dendritic cells. The data demonstrate that PFNA decreases the ratio of $\mathrm{CD} 19^{+}$cells within the splenocyte population in female mice (Figure 6). Although not statistically significant, PFNA tended to decrease the ratio of $\mathrm{CD} 19^{+}$cells in male mice as well. In addition, PFNA decreased the ratio of $\mathrm{CD} 14^{+}$cells within the splenocyte population in both male and female mice (Figure 7).

\section{PFNA increases TNFa in response to LPS}

Because $\mathrm{CD} 14^{+}$cells in spleen appeared to be particularly sensitive to PFNA as shown in Figure 7, we investigated the effect of PFNA on induction of TNFa in LPS-treated mice, a response mediated in large part by $\mathrm{CD}_{1}{ }^{+}$cells. Interestingly, PFNA administration caused a marked increase in TNFa production in LPS-treated mice, which was an unexpected finding (Figure 8).

\section{Discussion}

The present studies are the first to demonstrate that acute exposure to PFNA results in severe immunotoxicity in both male and female C57BL/6 mice. Specifically, the current studies show that a single dose of PFNA $(0.1 \mathrm{mmol} / \mathrm{kg})$ substantially decreases spleen size in male and female mice, which correlates with markedly decreased numbers of RBCs and leukocytes in spleen. Furthermore, the sensitivity of various leukocyte populations to PFNA is not uniform. In thymus, PFNA toxicity is most evident in the $\mathrm{CD} 4^{+} \mathrm{CD} 8^{+}$double-positive population, which represents the most rapidly dividing population in thymus.

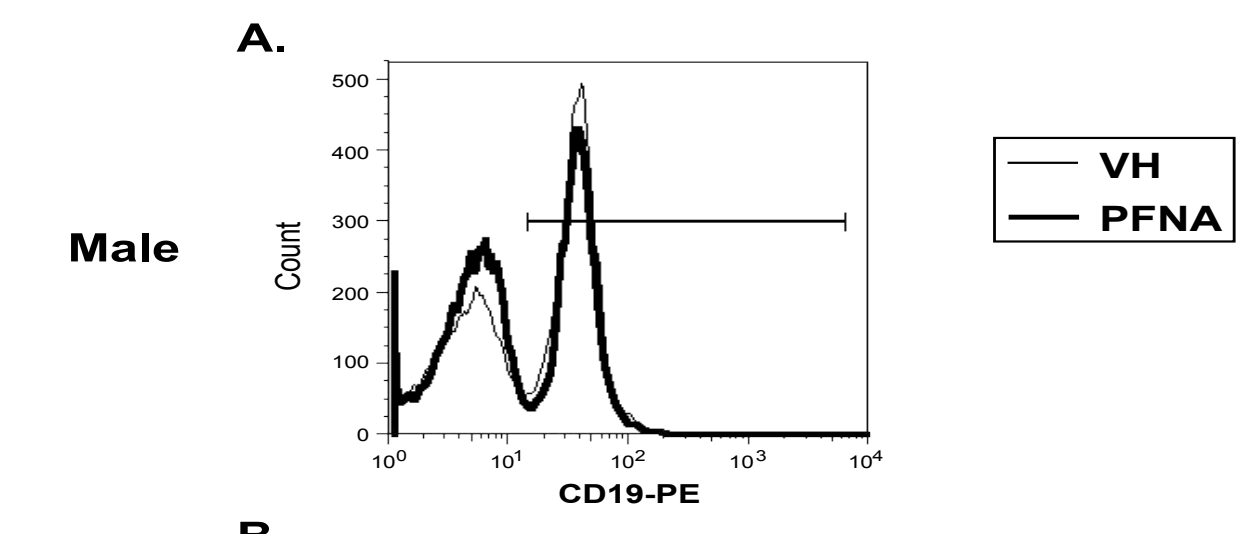

B.
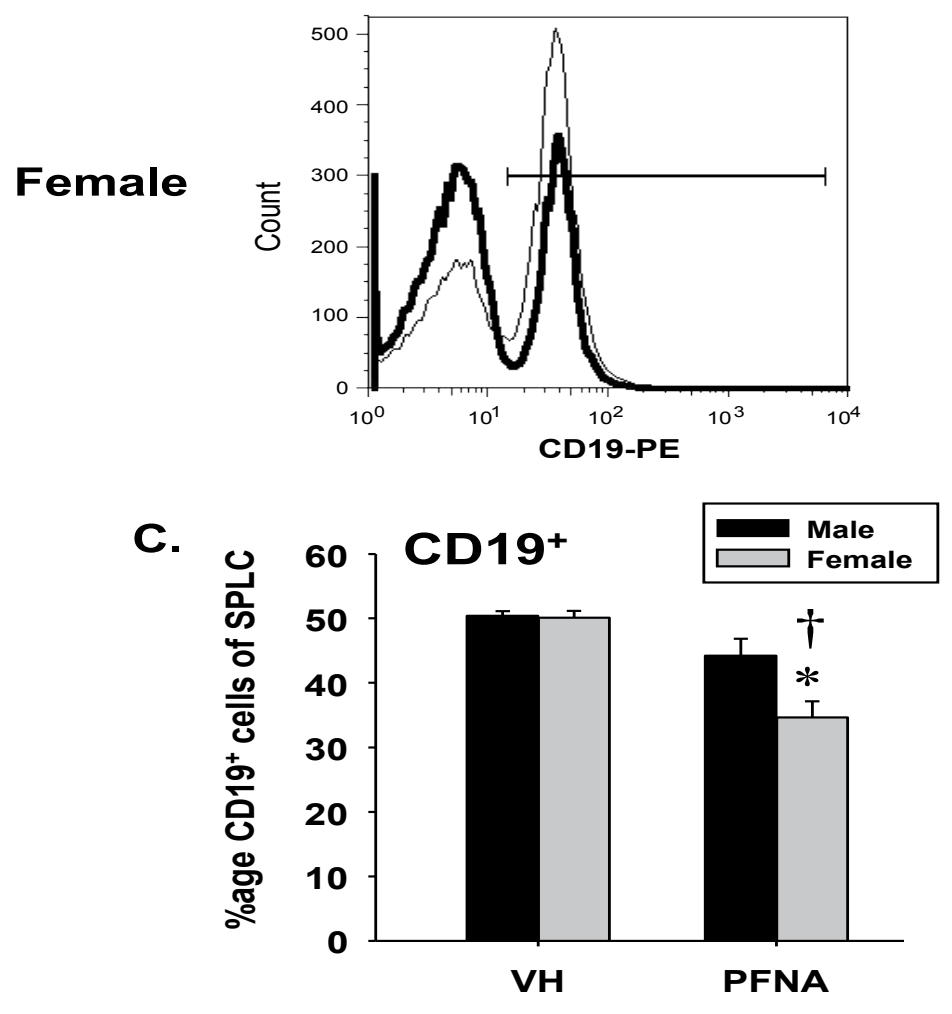

Figure 6: Decrease in the percentage of $\mathrm{CD} 19^{+}$cells in spleen of female PFNA-treated mice. Unfractionated splenocytes were washed, incubated with fluorochromeconjugated antibodies and analyzed by flow cytometry. A representative histogram of $\mathrm{CD} 19^{+} \mathrm{B}$ cells is depicted from $\mathrm{VH}$-treated (shown in thin gray line) and $\mathrm{PFNA}-$ treated (shown in heavy black line) $(A)$ male and (B) female mice. (C) Graphical representation of CD19+ $B$ cell percentage with respect to the whole splenocyte population. The cells were gated on those that were CD4 CD8. The data are presented as mean $\pm \mathrm{SE}$. ${ }^{*}$ denotes $\mathrm{p}<0.05$ as compared to the vehicle $(\mathrm{VH})$ control for the same gender. $\dagger$ denotes $p<0.05$ as compared between genders for the same treatment group. 


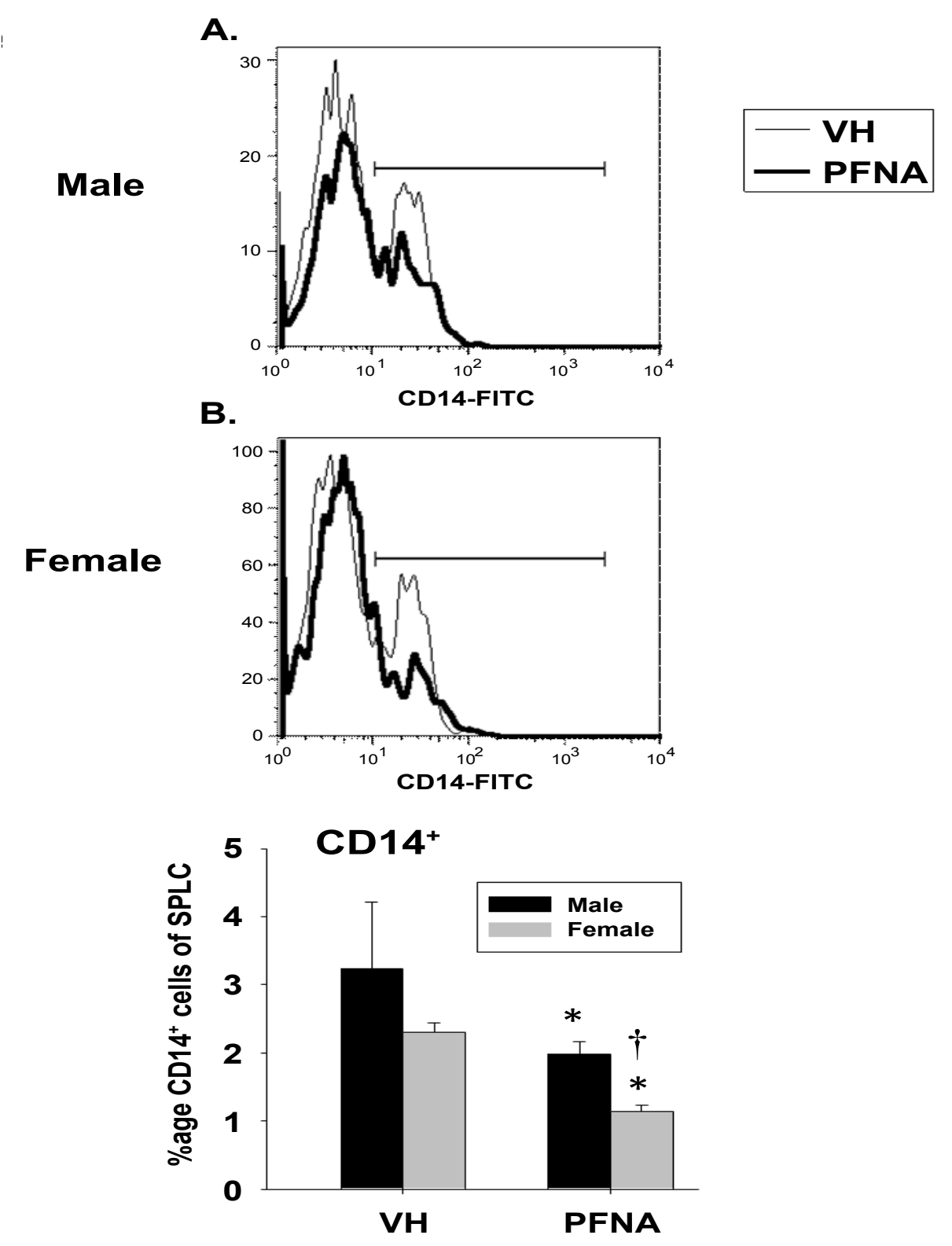

Figure 7: Decrease in the percentage of $\mathrm{CD} 14^{+}$cells in spleen of male and female PFNA-treated mice. Unfractionated splenocytes were washed, incubated with fluorochrome-conjugated antibodies and analyzed by flow cytometry. The cells are gated on the CD4-CD8-CD19- population. A representative histogram of CD14 cells is depicted from VH-treated (shown in thin gray line) and PFNA-treated (shown in heavy black line) (A) male and (B) female mice. (C) Graphical representation of $\mathrm{CD} 14^{+}$cell percentage with respect to the whole splenocyte population. The cells were gated on those that were CD4-CD8-CD19-. The data are presented as mean \pm SE. * denotes $p<0.05$ as compared to the vehicle $(\mathrm{VH})$ control for the same gender. $\dagger$ denotes $p<0.05$ as compared between genders for the same treatment group.

Interestingly, a marked increase in TNFa production in response to LPS was observed in PFNA-treated mice compared to vehicle controls. Overall, the current studies demonstrate decreased leukocyte viability in spleen and thymus as well as enhanced response to LPS by a single dose of PFNA in C57BL/6 mice.

The pronounced decrease in lymphoid organ weight and leukocyte numbers by PFNA is strikingly similar to the effects of other PFCs, such as PFOA. Like PFNA, PFOA administration causes considerable splenic atrophy and a concomitant decrease in the number of splenocytes [13]. In addition, the $\mathrm{CD} 4^{+} \mathrm{CD}^{+}$double-positive population in thymus is more sensitive to PFOA than other thymic populations, which is consistent with the effects of PFNA (Figure 5). PFOA also diminishes immune function as assessed by IgM production in the plaque-forming colony assay $[14,15]$. In contrast to PFNA and PFOA, PFOS does not decrease spleen and thymus weights in Sprague-Dawley rats or B6C3F1 mice $[16,17]$. The current studies in male and female C57BL/6 mice are consistent with previous observations of toxicity in lymphoid organs by PFNA in male Sprague-Dawley rats and male BALB/C mice $[12,18,19]$. Collectively, the present studies indicate that PFNAmediated immunotoxicity resembles that of other PFCs, in particular, PFOA.

Previous studies indicate that a number of PFCs, including PFOA and PFOS, cause peroxisome proliferation in hepatocytes, which is associated with activation of peroxisome proliferator-activated receptor 


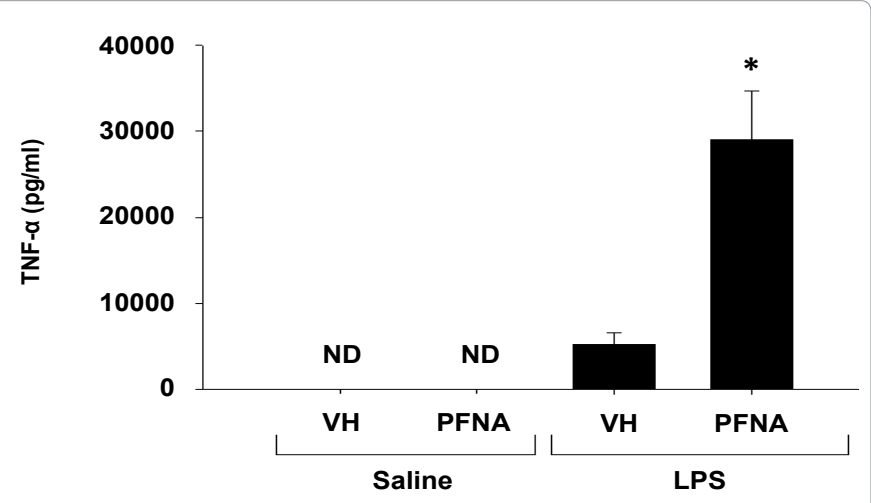

Figure 8: Increase in LPS-induced serum TNFa in PFNA-treated male mice. Blood was collected from PFNA-treated mice $1 \mathrm{hr} 30 \mathrm{~min}$ after they were injected with $1 \mathrm{mg} / \mathrm{kg}$ LPS or saline and serum TNFa levels were analyzed by ELISA. The data are presented as mean \pm SE. ND denotes samples that were below the level of detection of the assay. ${ }^{*}$ denotes $p<0.05$ as compared to the vehicle $(\mathrm{VH})$ control.

alpha (PPARa) [20-22]. Of importance to the current studies, PPARa agonists have been shown to have a number of immunomodulatory effects [6]. Administration of the synthetic PPARa agonist, Wy-14,643, decreased spleen weight and splenic cellularity as well as thymus weight and thymic cellularity in C57BL/6 mice [23]. Furthermore, PFOA exposure decreased spleen weight and splenic cellularity, in wild-type, but not PPARa-null mice. The decreased thymus weight and cellularity by PFOA was attenuated, but not eliminated, in PPAR $\alpha$-null mice, suggesting the effects in thymus are partially mediated through PPARa. The role of PPARa in PFOA-mediated toxicity remains controversial, however, because hepatomegaly by PFOA is observed in PPARa-null mice, suggesting this effect is independent of PPARa [23]. Further studies are needed to determine the role of PPAR $\alpha$ in PFNA-mediated immunotoxicity.

The enhanced TNFa response to LPS in PFNA-treated mice was an unexpected finding. Because there was significant splenic and thymic atrophy and decreased leukocyte viability in PFNA-treated mice, we initially hypothesized that PFNA would decrease the TNFa response to LPS. The marked increase in TNFa production in response to LPS by PFNA suggests that PFNA treatment primes CD14 $4^{+}$phagocytes for an inflammatory response. This may due to the marked decrease in leukocyte viability in PFNA-treated animals. Cell death, in particular necrosis, triggers inflammatory response or priming of macrophages and other phagocytes and thus could cause an enhanced TNFa response to LPS.

The average serum concentration of PFNA in the U.S. population is approximately $1.5 \mathrm{ng} / \mathrm{ml}[24,25]$. However, it is important to note that there is significant interindividual variability in PFNA serum levels, ranging from 5 to 50 times higher than the mean, due to numerous factors, such as age, race and gender, among other factors [24,26]. It has also been reported that populations living near PFC manufacturing facilities have significantly higher PFC blood concentrations than the national mean in the U.S., which reach as high as 300 times greater than average in certain communities [27]. Occupational exposure of workers within the manufacturing facility is potentially even higher. Although an epidemiological study on the effects of occupational PFNA exposure on blood lipids and liver enzyme activity has been published, the blood concentrations of PFNA in exposed workers was not reported in this study [28]. Given that PFNA blood concentrations have recently doubled over the course of 6 years in the U.S., all of these factors are of potential concern [29]. Indeed, given that PFNA blood concentrations in other industrialized countries are reported to be higher than in the U.S., PFNA is a matter of global concern [30]. Our focus has been on the effects of a single high dose exposure to PFNA, which is potentially relevant to inadvertant high dose occupational exposure as well as to sensitive populations in which PFNA blood concentrations are higher than the mean or a combination of both of these factors.

Collectively, the findings of the present studies demonstrate severe immunotoxic effects from a single dose of PFNA, including marked lymphoid organ atrophy, splenic and thymic cellularity, and altered ratios of leukocyte populations. Further investigation will be needed in order to better predict the consequences of acute PFNA exposure on immune organs and immune cellularity in humans.

\section{Acknowledgement}

The authors would like to acknowledge Dr. Joyce Slusser and Mr. Michael Stewart for technical assistance with flow cytometry analyses, the members of the Klaassen lab for careful reading and editing of the manuscript and Dr. Bryan Copple for use of instrumentation and reagents. This work was supported by the National Institutes of Health grants: T32 ES007079, R01 ES013714, and P20 RR021940 (to C.D.K.) and K99 ES018885 (to C.E.R).

\section{References}

1. Fromme H, Tittlemier SA, Völkel W, Wilhelm M, Twardella D (2009) Perfluorinated compounds--exposure assessment for the general population in Western countries. Int J Hyg Environ Health 212: 239-270.

2. Vestergren R, Cousins IT (2009) Tracking the pathways of human exposure to perfluorocarboxylates. Environ Sci Technol 43: 5565-5575.

3. Lau C, Butenhoff JL, Rogers JM (2004) The developmental toxicity of perfluoroalkyl acids and their derivatives. Toxicol Appl Pharmacol 198: 231241.

4. Prevedouros K, Cousins IT, Buck RC, Korzeniowski SH (2006) Sources, fate and transport of perfluorocarboxylates. Environ Sci Technol 40: 32-44

5. GL Kennedy, Butenhoff JL, Olsen GW, O'Connor JC, Seacat AM, et al. (2004) The toxicology of perfluorooctanoate. Crit Rev Toxicol 34: 351-384.

6. DeWitt JC, Shnyra A, Badr MZ, Loveless SE, Hoban D, et al. (2009) Immunotoxicity of perfluorooctanoic acid and perfluorooctane sulfonate and the role of peroxisome proliferator-activated receptor alpha. Crit Rev Toxico 39: 76-94

7. Wolf CJ, Zehr RD, Schmid JE, Lau C, Abbott BD (2010) Developmenta effects of perfluorononanoic Acid in the mouse are dependent on peroxisome proliferator-activated receptor-alpha. PPAR Res 2010.

8. Feng Y, Fang X, Shi Z, Xu M, Dai J (2010) Effects of PFNA exposure on expression of junction-associated molecules and secretory function in rat Sertoli cells. Reprod Toxicol 30: 429-437.

9. Feng Y, Shi Z, Fang X, Xu M, Dai J (2009) Perfluorononanoic acid induces apoptosis involving the Fas death receptor signaling pathway in rat testis. Toxicol Lett 190: 224-230.

10. Eriksen KT, Raaschou-Nielsen $O$, Sørensen $M$, Roursgaard M, Loft $S$, et al (2010) Genotoxic potential of the perfluorinated chemicals PFOA, PFOS, PFBS, PFNA and PFHxA in human HepG2 cells. Mutat Res 700: 39-43.

11. Nelson JW, Hatch EE, Webster TF (2010) Exposure to polyfluoroalkyl chemicals and cholesterol, body weight, and insulin resistance in the general U.S. population. Environ Health Perspect 118: 197-202.

12. Fang $X$, Zhang L, Feng $Y$, Zhao $Y$, Dai J (2008) Immunotoxic effects of perfluorononanoic acid on BALB/c mice. Toxicol Sci 105: 312-321.

13. Yang Q, Xie Y, Depierre JW (2000) Effects of peroxisome proliferators on the thymus and spleen of mice. Clin Exp Immunol 122: 219-226.

14. Yang Q, Abedi-Valugerdi M, Xie Y, Zhao XY, Möller G, et al. (2002) Potent suppression of the adaptive immune response in mice upon dietary exposure to the potent peroxisome proliferator, perfluorooctanoic acid. Int Immunopharmacol 2: 389-397.

15. Dewitt JC, Copeland CB, Strynar MJ, Luebke RW (2008) Perfluorooctanoic 
Citation: Rockwell CE, Turley AE, Cheng X, Fields PE, Klaassen CD (2013) Acute Immunotoxic Effects of Perfluorononanoic Acid (PFNA) in C57BL/6 Mice. Clin Exp Pharmacol S4: 002. doi:10.4172/2161-1459.S4-002

Page 9 of 9

acid-induced immunomodulation in adult C57BL/6J or C57BL/6N female mice. Environ Health Perspect 116: 644-650.

16. Peden-Adams MM, Keller JM, Eudaly JG, Berger J, Gilkeson GS, et al. (2008) Suppression of humoral immunity in mice following exposure to perfluorooctane sulfonate. Toxicol Sci 104: 144-154.

17. DE Lefebvre, I Curran, C Armstrong, L Coady, M Parenteau, et al. (2008) Immunomodulatory effects of dietary potassium perfluorooctane sulfonate (PFOS) exposure in adult Sprague-Dawley rats. J Toxicol Environ Health A 71: $1516-1525$

18. Fang X, Feng Y, Shi Z, Dai J (2009) Alterations of cytokines and MAPK signaling pathways are related to the immunotoxic effect of perfluorononanoic acid. Toxicol Sci 108: 367-376.

19. Fang X, Feng Y, Wang J, Dai J (2010) Perfluorononanoic acid-induced apoptosis in rat spleen involves oxidative stress and the activation of caspaseindependent death pathway. Toxicology 267: 54-59.

20. Intrasuksi U, Rangwala SM, O'Brien M, Noonan DJ, Feller DR (1998) Mechanisms of peroxisome proliferation by perfluorooctanoic acid and endogenous fatty acids. Gen Pharmacol 31: 187-197.

21. Vanden Heuvel JP, Thompson JT, Frame SR, Gillies PJ (2006) Differential activation of nuclear receptors by perfluorinated fatty acid analogs and natural fatty acids: a comparison of human, mouse, and rat peroxisome proliferatoractivated receptor-alpha, -beta, and -gamma, liver X receptor-beta, and retinoid $X$ receptor-alpha. Toxicol Sci 92: 476-489.

22. Berthiaumeand J, KB Wallace (2002) Perfluorooctanoate, perflourooctanesulfonate, and $\mathrm{N}$-ethyl perfluorooctanesulfonamido ethanol; peroxisome proliferation and mitochondrial biogenesis. Toxicol Lett 129: 23-32.

23. Yang Q, Xie Y, Alexson SE, Nelson BD, DePierre JW (2002) Involvement of the peroxisome proliferator-activated receptor alpha in the immunomodulation caused by peroxisome proliferators in mice. Biochem Pharmacol 63: 18931900.

24. CDC (2012) The Fourth National Report on Human Exposure to Environmenta Chemicals, National Report on Human Exposureto Environmental Chemicals Centers of Disease Control, Department of Health and Human Services.

25. CDC (2009) The Fourth National Report on Human Exposure to Environmenta Chemicals, National Report on Human Exposure to Environmental Chemicals, CDC, Department of Health and Human Services.

26. Schecter A, Malik-Bass N, Calafat AM, Kato K, Colacino JA, et al. (2012) Polyfluoroalkyl compounds in Texas children from birth through 12 years of age. Environ Health Perspect 120: 590-594.

27. Frisbee SJ, Brooks AP Jr, Maher A, Flensborg P, Arnold S, et al. (2009) The C8 health project: design, methods, and participants. Environ Health Perspect 117: $1873-1882$

28. Mundt DJ, Mundt KA, Luippold RS, Schmidt MD, Farr CH (2007) Clinica epidemiological study of employees exposed to surfactant blend containing perfluorononanoic acid. Occup Environ Med 64: 589-594.

29. Calafat AM, Wong LY, Kuklenyik Z, Reidy JA, Needham LL (2007) Polyfluoroalkyl chemicals in the U.S. population: data from the National Health and Nutrition Examination Survey (NHANES) 2003-2004 and comparisons with NHANES 1999-2000. Environ Health Perspect 115: 1596-1602.

30. Takagi S, Yoshida J, Adachi F (2012) Concentrations of Perfluorinated Compounds in Tap Water and Human Serum from Osaka, Japan. Interdisciplinary Studies on Environmental Chemistry-Environmental Pollution and Ecotoxicology 6: 245-251.
This article was originally published in a special issue, Novel developments in Immunopharmacology and Immunotoxicology handled by Editor(s). Dr. Yuliang Ma, Weill Cornell Medical College, USA 